\title{
Modeling spike-count dependence structures with multivariate Poisson distributions
} Arno Onken*1,2 and Klaus Obermayer ${ }^{1,2}$

Address: ${ }^{1}$ Bernstein Center for Computational Neuroscience, Berlin, 10115, Germany and ${ }^{2}$ Department of Electrical Engineering and Computer Science, Berlin Institute of Technology, Berlin, 10587, Germany

Email: Arno Onken* - aonken@cs.tu-berlin.de

* Corresponding author

from Seventeenth Annual Computational Neuroscience Meeting: CNS*2008 Portland, OR, USA. 19-24 July 2008

Published: II July 2008

BMC Neuroscience 2008, 9(Suppl I):PI27 doi:10.1 186/I47I-2202-9-SI-PI27

This abstract is available from: http://www.biomedcentral.com/I47I-2202/9/SI/PI 27

(c) 2008 Onken and Obermayer; licensee BioMed Central Ltd.

Previous studies use multivariate Gaussian distributions as models of correlated spike-counts [1]. However, this approximation is not appropriate for short time intervals and fails to model realistic dependence structures. To eradicate these shortcomings, we propose alternative joint distributions that are marginally Poisson distributed and contain a broad range of dependence structures for count variables.

We apply two methods to generate multivariate Poisson distributions of dependent spike-counts. The first approach employs sums of hidden variables to introduce dependencies between the Poisson distributed counts [2]. The second approach introduces dependencies by means of copulas of several classes. Copulas are functions that couple marginal cumulative distribution functions to form a joint distribution function with the same margins [3].

The methods are evaluated on a data set of simultaneously measured spike-counts on $100 \mathrm{~ms}$ intervals of up to three neurons in macaque MT responding to stochastic dot stimuli [4]. Parameters are estimated by the inference-for margins method: first the margin likelihoods are sepa-
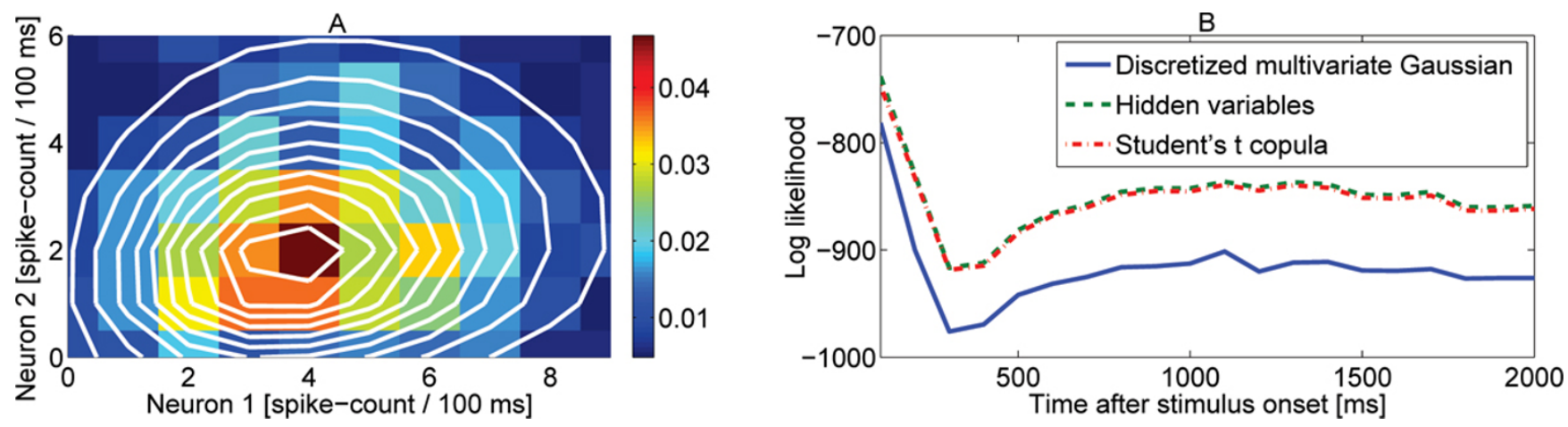

\section{Figure I}

Likelihoods of the distribution fits to data from [4]. A. Empirical fractions of measured spike-counts for a representative neuron pair and white contour lines of the bivariate Poisson distribution with hidden variables. B. Log likelihoods for the discretized Gaussian, the multivariate Poisson distribution with hidden variables, and the best fitting copula at consecutive 100 ms time intervals after stimulus onset. 
rately maximized and then the coupling parameters are estimated given the parameterized margins. Resulting parameters are close to the maximum likelihood estimation with the advantage that the approach is also tractable for high dimensions. Goodness-of-fit is evaluated by cross-validation for the likelihoods. We find that a multivariate Poisson distribution with hidden variables provides the best overall fit to the data from [4] (see Figure 1).

\section{Acknowledgements}

This work was supported by BMBF grant 0IGQ04I0.

\section{References}

I. Abbott LF, Dayan P: The effect of correlated variability on the accuracy of a population code. Neural Computation 1999, I I:9I-10I.

2. Kawamura K: The structure of the multivariate Poisson distribution. Kodai Mathematical Journal 1979, 2:337-345.

3. Nelsen RB: An Introduction to Copulas New York: Springer-Verlag; 1998.

4. Zohary E, Newsome WT: Responses of pairs of neurons in macaque MT/V5 as a function of motion coherence in stochastic dot stimuli. Neural Signal Archive, nsa2004.2 [http:// www.neuralsignal.org].
http.//Www.biomedcentral.com/1471-2202/9/S1/P127 